\title{
A Collisional-Radiative Model for Hydrogen Atom Including Velocity Changing Collisions*)
}

\author{
Keisuke FUJII, Taiichi SHIKAMA, Keiji SAWADA ${ }^{1)}$, Motoshi GOTO $^{2)}$, Shigeru MORITA ${ }^{2)}$ and \\ Masahiro HASUO \\ Department of Mechanical Engineering and Science, Graduate School of Engineering, \\ Kyoto University, Kyoto 606-8501, Japan \\ ${ }^{1)}$ Department of Applied Physics, Faculty of Engineering, Shinshu University, Nagano 380-8553, Japan \\ ${ }^{2)}$ National Institute for Fusion Science, Toki 509-5292, Japan
}

(Received 7 December 2010 / Accepted 24 June 2011)

\begin{abstract}
We develop a collisional-radiative model for calculating the velocity distributions of excited hydrogen atoms. In the model, the velocity distributions are approximated using linear combinations of several basis functions which are treated as discrete velocity states, and only charge exchange collisions with proton are considered as velocity changing collisions. The populations in the velocity and excited states are calculated under an assumption of a quasi-stationary state. With the model we derive the velocity distribution of the ground state atoms from that of excited atoms in the $n=3$ level observed for an LHD plasma, where $n$ is the principal quantum number. The mean kinetic energy of the ground state atoms is estimated to be $5.6 \mathrm{eV}$, which is $0.05 \mathrm{eV}$ less than that of the $n=$ 3 atoms
\end{abstract}

(c) 2011 The Japan Society of Plasma Science and Nuclear Fusion Research

Keywords: collisional-radiative model, hydrogen atom, velocity distribution, charge exchange collision, velocity changing collision, LHD plasma, spectroscopy

DOI: $10.1585 /$ pfr.6.2401125

\section{Introduction}

In magnetically confined plasmas, dynamics of neutral atoms around the confined region is an energetically studied topic to improve plasma confinement. Line shape measurements of atomic hydrogen emissions have provided information about velocity distributions of the atoms. J. D. Hey et al. measured the Balmer series line shapes in TEXTOR [1]. They found that the velocity distributions of the excited hydrogen or deuterium atoms were in non-thermal. They attributed the high velocity atoms to be generated through charge exchange collisions with high velocity protons. Such non-thermal velocity distributions of hydrogen or deuterium atoms have also been observed in other devices, i.e., deuterium line profiles measured by $\mathrm{H}$. Kubo in JT-60U [2], hydrogen line shapes by T. Shikama in TRIAM-1 M [3] and by A. Iwamae in LHD (Large Helical Device) [4].

In our previous report [5], we have shown that the velocity distributions of excited hydrogen atoms in $n=3$, 4 and 5 levels, which were converted from the observed Balmer line profiles for the LHD plasma, are the same within the experimental accuracy, where $n$ is the principal quantum number. However, up to now, no models have been proposed to discuss relations among the velocity distributions of the excited and ground state atoms.

author's e-mail: fujii.keisuke@fs4.ecs.kyoto-u.ac.jp

*) This article is based on the presentation at the 20th International Toki Conference (ITC20).
In this work, we develop a collisional-radiative model to calculate the velocity distributions of the excited and ground state atoms.

\section{A Collisional-Radiative Model for Hydrogen Atoms Including the Ve- locity Distribution Function}

We consider a rate equation for a population in an excited state $p$ having velocity between $v$ and $v+\mathrm{d} v$ as

$$
\begin{aligned}
& \frac{\partial}{\partial t} N_{p}(v) \mathrm{d} v= \\
& \quad \sum_{q} \int \mathrm{d} v^{\prime} \underset{q \rightarrow p}{r}\left(v^{\prime} \rightarrow v\right) N_{q}\left(v^{\prime}\right) \mathrm{d} v \\
& \quad-\sum_{q} \int \mathrm{d} v^{\prime} \underset{p \rightarrow q}{r}\left(v \rightarrow v^{\prime}\right) N_{p}(v) \mathrm{d} v,
\end{aligned}
$$

where $q$ indicates an atomic or ionic state. $r_{q \rightarrow p}\left(v^{\prime} \rightarrow v\right)$ is the population transfer rate from state $q$ to $p$ in which the velocity is changed from $v^{\prime}$ to $v\left(p=q\right.$ or $v^{\prime}=v$ case is included). In the right hand side of equation (1), the first term indicates the population influx to $p$ state having velocity of $v \sim v+\mathrm{d} v$, while the second term indicates the population outflux.

In this work, we focus the discussion on a hydrogen plasma under a condition of $n_{\mathrm{e}}=n_{\mathrm{p}} \gg n_{\mathrm{H}}$, where $n_{\mathrm{e}}$, $n_{\mathrm{p}}$ and $n_{\mathrm{H}}$ are the electron, proton and atom densities, respectively, and containing no molecule. Since the velocity change by atomic collisions is negligible in this case, the 
rate $r_{q \rightarrow p}\left(v^{\prime} \rightarrow v\right)$ can be written as

$$
\begin{aligned}
& \underset{q \rightarrow p}{r}\left(v^{\prime} \rightarrow v\right)= \\
& \quad \underset{q \rightarrow p}{A} \delta\left(v^{\prime}-v\right)+\underset{q \rightarrow p}{C_{\mathrm{ex}}^{\mathrm{e}}} \delta\left(v^{\prime}-v\right) n_{\mathrm{e}} \\
& \quad+\underset{q \rightarrow p}{C_{\mathrm{ex}}^{\mathrm{p}}} \delta\left(v^{\prime}-v\right) n_{\mathrm{p}}+\underset{q \rightarrow p}{C_{\mathrm{cx}}^{\mathrm{p}}}\left(v^{\prime} \rightarrow v\right) n_{\mathrm{p}},
\end{aligned}
$$

where $A_{q \rightarrow p}$ is Einstein's A coefficient, $C_{\mathrm{ex} q \rightarrow p}^{e}$ is the transition rate coefficient by electron collisions, $C_{\mathrm{ex}}^{p}{ }_{q \rightarrow p}$ is that by proton collisions without velocity change. $\delta\left(v^{\prime}-v\right)$ is the delta function and $C_{c x q \rightarrow p}^{p}\left(v^{\prime} \rightarrow v\right)$ is the charge exchange rate coefficient by proton collisions, which is accompanied by the velocity change.

For the sake of simplicity in the calculation, we approximate $N_{p}(v)$ by a linear combination of several normalized Maxwell distribution functions $\phi_{i}(v)$ with coefficients $c_{p, i}$ as

$$
N_{p}(v)=\sum_{i} c_{p, i} \phi_{i}(v)
$$

The coefficient $c_{p, i}$ indicates the population in atomic state $p$ and velocity state $i$. Substitution of equation (3) into equation (1) gives a relation

$$
\frac{\partial}{\partial t} c_{p, i}=\sum_{q, j} \underset{q, j \rightarrow p, i}{r} c_{q, j}-\sum_{q, j} \underset{p, i \rightarrow q, j}{r} c_{p, i},
$$

where $r_{p, i \rightarrow q, j}$ is the population transfer rate between the atomic or ionic states and the velocity states calculated from the rates defined in equation (2). We use a quasistationary state approximation [6] except for the ground and ionic states, i.e., the left hand side of equation (4) is approximated to be zero for all the excited states. Under this approximation, the set of the equations can be written in a form

$$
c_{p, i}=\sum_{j} R_{p, i, j}^{\text {proton }} c_{\text {proton }, j}+\sum_{j} R_{p, i, j}^{1} c_{1, j},
$$

where $c_{\text {proton, } j}$ and $c_{1, j}$ denote the populations in the velocity state $j$ of the protons and the ground state atoms, respectively. $R_{p, i, j}^{\text {proton }}$ and $R_{p, i, j}^{1}$ are the respective rate coefficients which are functions of $T_{\mathrm{e}}, T_{\mathrm{p}}, n_{\mathrm{e}}$, and $n_{\mathrm{p}}$.

We adopted the cross sections of electron collisions evaluated or compiled by I. Bray et al. [7], L. Johnson [8] and R. K. Janev et al. [9]. For the proton excitation or deexcitation collisions, we use the cross sections compiled by R. K. Janev [9]. The differential cross sections of the charge exchange collisions are necessary to calculate $r_{p, i \rightarrow q, j}$. However, only the total cross sections are available for the excited states. Since the forward scattering is known to be dominant in the charge exchange collisions [10], we assume that all the collisions are the forward scattering. We use the calculated total cross sections by C. Harel [11] and CAPTURE code [12] developed by V. Shevelko et al. Figure 1 shows the total cross sections for charge exchange collisions relevant to the $n=1,2,3,4$, and 5 states. The lower state has the smaller cross section.

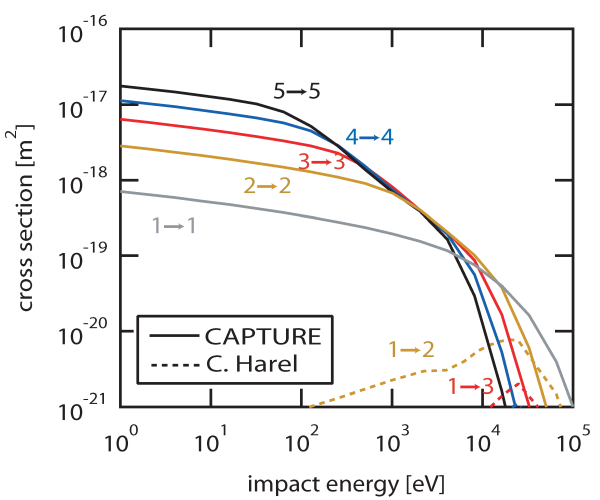

Fig. 1 Charge exchange cross sections calculated by C. Harel et $a l$. [11] and CAPTURE code [12]. The principal quantum numbers of the initial and final states are shown.

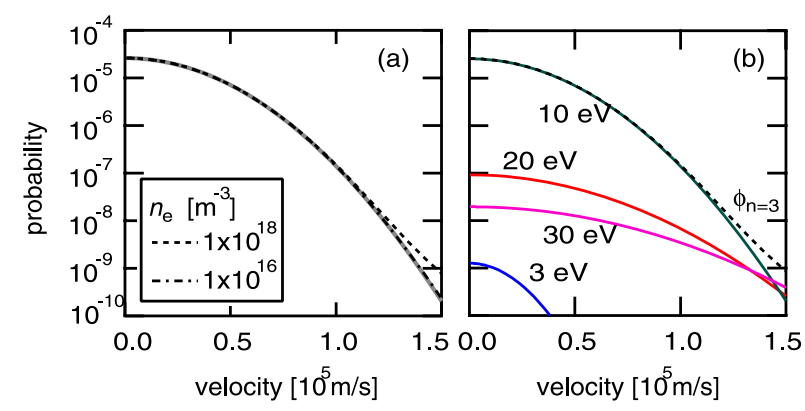

Fig. 2 (a) Calculated velocity distribution of $n=3$ atoms at $T_{\mathrm{e}}$ $=T_{\mathrm{p}}=20 \mathrm{eV}$ and $n_{\mathrm{e}}=n_{\mathrm{p}}=1 \times 10^{16} \mathrm{~m}^{-3}$ (dashed-dotted curve) and $1 \times 10^{18} \mathrm{~m}^{-3}$ (dashed curve). The areas are normalized to be unity. A Maxwellian velocity distribution with a temperature of $10 \mathrm{eV}$ is assumed for the ground state atoms, which is shown by the gray bold curve. (b) The calculated velocity distribution at $n_{\mathrm{e}}=n_{\mathrm{p}}$ $=1 \times 10^{18} \mathrm{~m}^{-3}$ and its velocity components. It is noticed that the population in the velocity state of $1 \mathrm{eV}$ is so small that it is not seen in the figure.

It is noted again that equation (5) is only for the excited states and we neglect the velocity change of the ground state atoms. This model can be applied to the system in which the charge exchange collision for the ground state is negligible.

In Fig. 2 (a), we show an example of the calculated velocity distribution of the excited atoms in the $n=3$ level assuming that the velocity distribution of the ground state atoms is expressed by a single Maxwellian function at a temperature of $10 \mathrm{eV}$ (the gray bold curve in Fig. 2 (a)). We assume an ionizing plasma, where the first term of the right hand side of equation (5) is negligible [6]. We use five Maxwellian functions at atomic temperatures of 1, 3, 10,20 and $30 \mathrm{eV}$ for $\phi_{i}(v)$. The electron and proton temperatures are assumed to be $T_{\mathrm{e}}=T_{\mathrm{p}}=20 \mathrm{eV}$. We set $n_{\mathrm{e}}=n_{\mathrm{p}}=1 \times 10^{16} \mathrm{~m}^{-3}$ (the dashed-dotted line in Fig. 2 (a)) and $1 \times 10^{18} \mathrm{~m}^{-3}$ (the dashed line). For the first case, the calculated velocity distribution of the $n=3$ atoms is almost the same as that of the ground state atoms, so that the 
dashed-dotted line and the gray bold line cannot be distinguished in Fig. 2 (a). For the latter case, the velocity distributions of the $n=3$ atoms have a high velocity tail. Figure 2 (b) shows the calculated velocity distribution for the $n=3$ atoms at $n_{\mathrm{e}}=n_{\mathrm{p}}=1 \times 10^{18} \mathrm{~m}^{-3}$ together with the fractions of five velocity functions. The contribution of the functions with the temperature higher than $T_{\mathrm{p}}$ is seen. This contribution is due to the positive dependence of the charge exchange rate coefficient on $T_{\mathrm{p}}$, which originates from the nearly flat energy dependence of the cross section around $20 \mathrm{eV}$ as can be seen in Fig. 1 .

For the purpose of quantifying the difference in the velocity distributions, we calculate the mean kinetic energy difference defined here as

$$
\Delta E_{p}=E_{p}-E_{1},
$$

where $E_{\mathrm{p}}$ is the mean kinetic energy of atoms in $p$ state and $E_{1}$ is that in the ground state.

We show $n_{\mathrm{e}}$ dependence of $\Delta E_{3}, \Delta E_{4}$, and $\Delta E_{5}$ in Fig. 3. Here, since we consider an ionizing plasma, $\Delta E_{p}$ is generated dominantly by the following two processes; 1 . hot $p$ state atoms generated by charge exchange from cold $p$ state atoms, and 2. hot atoms generated by charge exchange collision in the other excited states and populated into the $p$ state.

In a low density case, the process 1 is dominant. In this case, $\Delta E_{p}$ is caused as a result of the charge exchange collision during the lifetime of the $p$ state which is determined by the inverse of Einstein's A coefficients. The reason for that the higher state has the larger $\Delta E_{p}$ is that the higher state has the larger cross section and the smaller $\mathrm{A}$ coefficient. In a higher density case, the lifetime of the $p$ state is determined by the electron collisional depopulation, and the probability to undergo the charge exchange collision during the lifetime has no dependence on the den-

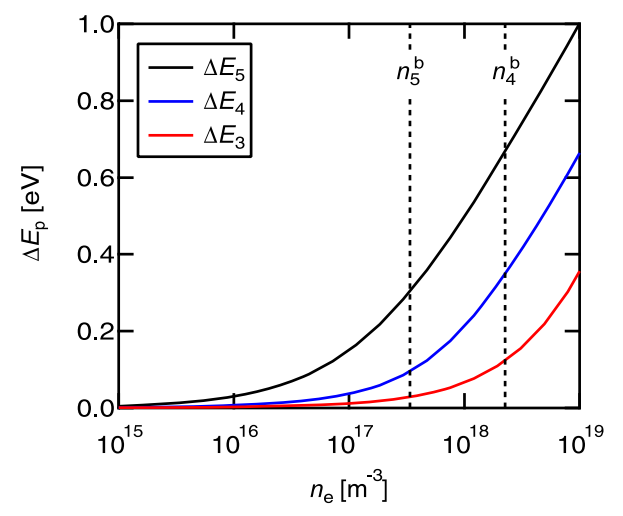

Fig. $3 \Delta E_{\mathrm{p}}$ for $n=3$ (red curve), 4 (blue curve), and 5 (black curve) atoms are shown. The horizontal axis is $n_{\mathrm{e}}=n_{\mathrm{p}}$. The vertical dashed lines indicate the boundary densities for $n=4$ and 5 atoms which are defined in the text. All the results are calculated under the condition of $T_{\mathrm{e}}=T_{\mathrm{p}}=$ $20 \mathrm{eV}$ and a single Mexwellian velocity distribution of the ground state atoms at a temperature of $10 \mathrm{eV}$. sity. Boundary densities $n_{p}^{\mathrm{b}}$ for the $n=4$ and 5 states, where the electron collisional depopulation rate equals to the $\mathrm{A}$ coefficient, are shown by the vertical dashed lines in Fig. 3. $\Delta E_{p}$ increases over $n_{p}^{\mathrm{b}}$ because of the process 2 , and it reflects the increase of the probabilities to have the charge exchange collisions in the other states.

For estimating the plasma condition to which this model can be applied, we consider a plasma with a size of $0.1 \mathrm{~m}$. It takes $2 \times 10^{-6} \mathrm{~s}$ for the ground state atoms having a temperature of $10 \mathrm{eV}$ to pass through the plasma (the most probable velocity is $4 \times 10^{4} \mathrm{~m} / \mathrm{s}$ ). The charge exchange rate coefficient for the ground state atoms then becomes $2 \times 10^{-14} \mathrm{~m}^{3} / \mathrm{s}$ when the ion temperature is $20 \mathrm{eV}$. In a lower density plasma than $10^{19} \mathrm{~m}^{-3}$, the probability to undergo charge exchange collision is smaller than 0.3 , so that this model can be applied. For this reason, we plot $\Delta E_{p}$ in Fig. 3 for lower density than $10^{19} \mathrm{~m}^{-3}$.

\section{The Collisional-Radiative Model Analysis of the Observed Spectra in an LHD Peripheral Plasma}

As an example, we show the velocity distributions of the $n=3,4$ and 5 hydrogen atoms evaluated from the simultaneously observed Balmer- $\alpha,-\beta$, and $-\gamma$ spectra in an LHD plasma in Fig. 4 (a), (b) and (c), respectively, with the open circles. For the observation, a radial viewing chord passing through the peripheral region was chosen and the multi-wavelength-range fine-resolution spectrometer was

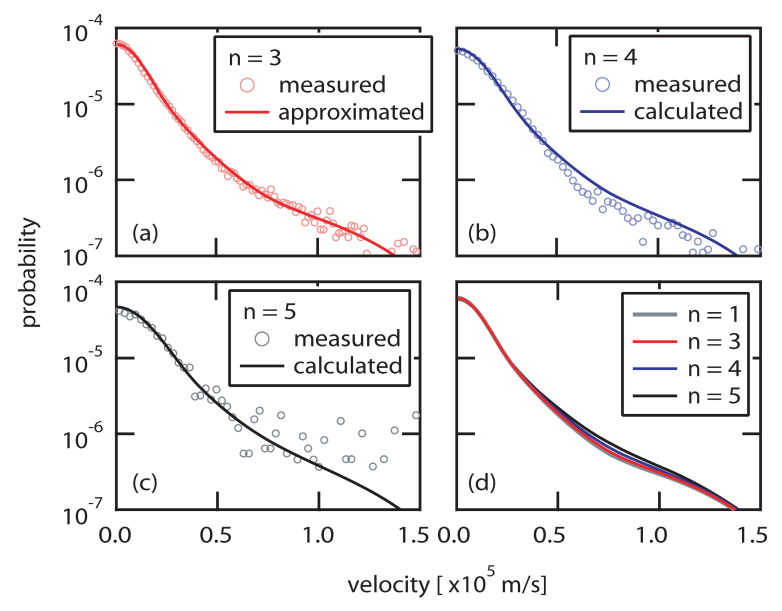

Fig. 4 Velocity distributions of hydrogen atoms in the $n=3$ (a), 4 (b), and 5 (c) levels converted from the observed spectra for LHD. The area is normalized to be 1 . The approximated velocity distribution of the $n=3$ atoms with the equation (3) is shown by the solid curve in (a). The calculated velocity distributions of the $n=4$ and 5 atoms are shown by the solid curves in (b) and (c), respectively. It is noted that the approximated and calculated velocity distributions are convoluted with the instrumental functions for the purpose of comparison with the observed ones. (d): Calculated velocity distributions of the $n=3,4$, and 5 atoms and the ground state atoms without the convolution. 
used [13]. It is noted that the velocity distributions shown in Fig. 4 (a)-(c) are the convoluted ones with the instrumental functions of the spectrometer. The FWHMs of the instrumental functions are $0.9 \times 10^{4} \mathrm{~m} / \mathrm{s}, 1.4 \times 10^{4} \mathrm{~m} / \mathrm{s}$ and $2.1 \times 10^{4} \mathrm{~m} / \mathrm{s}$ for the $n=3,4$ and 5 excited atoms respectively.

In the edge region of the LHD plasma, the plasma parameters have steep spatial gradients, and the relation between the spatial distribution and the observed velocity distribution of hydrogen atoms was discussed recently by Goto et al. [14]. In the present model, however, we neglect the spatial distribution of the plasma parameters and examine the effect of the velocity changing collisions on the atomic velocity distribution. We adopt $T_{\mathrm{e}}$ and $n_{\mathrm{e}}$ at the emission location as $20 \mathrm{eV}$ and $2 \times 10^{18} \mathrm{~m}^{-3}$, respectively, which are measured by Thomson scattering method [15]. Since the plasma consists of almost pure hydrogen, $n_{\mathrm{p}}$ is expected to be the same as $n_{\mathrm{e}}$. The atomic density there is estimated to be $1 \times 10^{16} \mathrm{~m}^{-3}$ from the emission intensity and the plasma parameters, which satisfies the condition of $n_{\mathrm{e}}=n_{\mathrm{p}} \gg n_{\mathrm{H}}$. We assume $T_{\mathrm{p}}=T_{\mathrm{e}}$.

We calculate the coefficients $R_{p, i, j}^{1}$ in equation (5) for each excited state. We fit the measured velocity distribution of the $n=3$ state with equation (3) and evaluate the populations $c_{1, j}$. The fitted result is shown in Fig. 4 (a) with the solid curve. The velocity distribution of the ground state and $n=4$ and 5 atoms are then calculated using equation (5). The calculated velocity distribution functions of the $n=4$ and 5 atoms are shown in Fig. 4 (b) and (c), respectively, with the solid curves. It is found that the calculated results well reproduce the experimental ones. In Fig. 4 (d) we compare the calculated velocity distributions of the $n=1,3,4$ and 5 atoms. The mean kinetic energy of the ground state atoms is $E_{1}=5.6 \mathrm{eV}$ and the kinetic energy differences $\Delta E_{p}$ are $0.05,0.2$ and $0.3 \mathrm{eV}$ for the $n$ $=3,4$ and 5 atoms, respectively.

\section{Conclusion}

We developed a collisional-radiative model for calculating the velocity distributions of hydrogen atoms. In this model, the velocity distributions of the atoms in the ground state and excited states are approximated by linear combinations of several basis functions and the populations in the atomic states and velocity states are derived under an assumption of a quasi-stationary state. From the comparison with the observed velocity distributions of excited hydrogen atoms in an LHD periphery plasma, the velocity distribution of the ground state atoms was derived.

\section{Acknowledgement}

This work was supported by the National Institute for Fusion Science (Grant No. NIFS08KOAP020). One of the authors (K. F.) would like to thank Dr. V. Shevelko for his remarks and calculations on the charge exchange cross sections.

[1] J.D. Hey, M. Korten, Y.T. Lie et al., Contrib. Plasma Phys. 36, 583 (1996).

[2] H. Kubo, H. Takenaga, T. Sugie et al., Plasma Phys. Control. Fusion 40, 1115 (1998).

[3] T. Shikama, S. Kado, H. Zushi et al., Plasma Phys. Control. Fusion 48, 1125 (2006).

[4] A. Iwamae, M. Hayakawa, M. Atake et al., Phys. Plasmas 12, 042501 (2005).

[5] K. Fujii, K. Mizushiri, T. Nishioka et al., Nucl. Instrum. Methods A 623, 690 (2010).

[6] T. Fujimoto, Plasma Spectroscopy, Oxford (2004).

[7] I. Bray and A.T. Stelbovics, Phys. Rev. A 46, 6995 (1992).

[8] L.C. Johnson, Astrophys. J. 174, 227 (1972).

[9] R.K. Janev, W.D. Langer, K. Evans Jr. et al., Elementary Processes in Hydergon-Helium Plasmas, Springer (1987).

[10] P.S. Krstic and D.R. Schultz, J. Phys. B 36, 385 (2003).

[11] C. Harel, H. Jouin and B. Pons, Atomic Data and Nuclear Tables 68, 279 (1998).

[12] V. Shevelko, D. Kato, M.Y. Song et al., Nucl. Instrum. Methods B 267, 3395 (2009).

[13] K. Fujii, K. Mizushiri, T. Nishioka et al., Rev. Sci. Instrum. 81, 033106 (2010).

[14] M. Goto, K. Sawada, K. Fujii, M. Hasuo and S. Morita, Nucl. Fusion 51, 023005 (2011).

[15] K. Narihara, I. Yamada, H. Hayashi and K. Yamauchi, Rev. Sci. Instrum. 72, 1122 (2001). 